\title{
The investigation of Pneumocystis jirovecii colonization in adult individuals of Turkish population
}

\begin{abstract}
Pneumocystis jirovecii known Pneumocystis carinii is the cause of opportunistic infections in the lower respiratory tract of immunocompromised hosts. It was considered one of the leading causes of morbidity and mortality among both HIV nonHIV patients. Pneumocystis colonization in the lower respiratory tract is considered a public health problem; since they play a role as a major reservoir of Pneumocystis jirovecii and a source of infection for susceptible subjects. In our prospective study, we aimed to investigate colonization prevalence and related risk factors among adult population in our region using combined samples collected from adults aged 18 years and above of three different groups: general adult population, health care workers and immunocompromised individuals. Samples were analyzed by real-time PCR targeting P. jirovecii MSG. DNA was detected in $(22.8 \%)$ of all participants, $(21 \%)$ of general adults, $(22 \%)$ of health care workers, $(28 \%)$ of immunocompromised individuals. Although the frequency of colonization in immunocompromised individuals was higher than other participants, it was not significant statistically. Colonization rate was found to be higher among the participants used antibiotic or immunosuppressive treatment; however, the difference is not statistically significant. Hospital durations of immunocompromised individuals didn't affect the colonization rate. However, the duration of underlying disease was longer in the immunocompromised participants colonized with P. jirovecii than non-colonized ones $(\mathrm{p}=0.001)$ !!
\end{abstract}

\author{
Special Issue - 2018
}

\author{
Qoraan I,' Oz Y, ${ }^{2}$ Metintas M, ${ }^{3}$ Durmaz G' \\ 'Department of Basic Medical Sciences, Arab American \\ University, Faculty of Dentistry, Plaestine \\ ${ }^{2}$ Department of Microbiology, Eskișehir Osmangazi University, \\ Turkey \\ ${ }^{3}$ Department of Public Health, Eskișehir Osmangazi University, \\ Turkey
}

Correspondence: Iman Qoraan, Department of Basic Medica Sciences, Arab American University Faculty of Dentistry, Jenin, Plaestine,Tel 09759509 1906, Email imanafaneh@yahoo.com

Received: October 28, 2017 | Published: July 31, 2018
Abbreviations: BAL, Bronchoalveolar Lavage; PCP, Pneumocystis Pneumonia; MSGC1, Major Surface Glycoprotein Subunit; mtLSU-rRNA, Pneumocystis Mitochondrial Large-Subunit Gene

\section{Introduction}

Pneumocystis jirovecii previously known Pneumocystis carinii is the cause of opportunistic infections in the lower respiratory tract of immunocompromised hosts mainly among patients with HIV infected, hematologic malignancies, congenital immunodeficiency, organ transplant recipients, and patients receiving immunosuppressive drugs. ${ }^{1}$ The organism was considered as one of the most leading causes of morbidity and mortality among HIV patients before the introduction of antiretroviral therapy. However, Pneumocystis pneumonia (PCP) is associated with significant mortality and morbidity rates among non-HIV patients. ${ }^{2}$ The mechanism of Pneumocystis transmission was debating. Although previously it was thought that PCP infection is a reactivation of latent infection acquired during childhood, recently it has been recognized to be de novo exposure from either environment or individuals with PCP or colonized with Pneumocystis. ${ }^{3}$ Pneumocystis colonization has been defined as the presence of $P$. jirovecii in respiratory specimens of persons without signs or symptoms of acute pneumonia ${ }^{3}$ Pneumocystis jirovecii can colonize in lower respiratory tract without causing signs and symptoms and PCP may develop in these colonized individuals. Thus, Pneumocystis colonized individuals could also be a problem for public health since they could play a role as a major reservoir of Pneumocystis jirovecii and a source of infection for susceptible subjects. ${ }^{4}$ Furthermore, patients at risk for PCP who receive long term anti-pneumocystis prophylactic treatments may be colonized with drug mutated pneumocystis species. In addition, colonization may stimulate a host inflammatory response leading to lung damage and progression of lung disease such as chronic obstructive pulmonary disease (COPD). ${ }^{1,3}$ Therefore, we planned a prospective study and aimed to investigate the colonization prevalence and related risk factors among adult population in our region.

\section{Materials and method}

\section{Participants}

We constituted three different study groups including general adult population, health care workers because of the high possibility of exposure to patients with PCP and immunocompromised individuals due to the high risk for PCP;

i. General adult population $(n=100)$ who had not been exposed to patients or a hospital environment within last one year;

ii. Health care workers $(n=100)$ who work for at least one year in various inpatient departments of Eskisehir Osmangazi University Tertiary Hospital; and

iii. Immunocompromised individuals who were mostly treated with corticosteroids and/or other immunosuppressive medications for any hematological disorder in Hematology Unit.

This study was carried out from February to May 2015. Written informed consent was obtained from all participants before samples collection. Exclusion criteria were positivity of HIV tests, presence of any clinical or radiological sign and symptoms for PCP, and children under 18 years old. For each participant, a standardized form was constituted to obtain some information including demographic characteristics, medical history, habits etc. The study was approved by the Clinical Research Ethics Committee of Uskudar University. 


\section{Samples}

To increase the diagnostic yield, both oral washing and nasal swab specimens were collected from all participants. ${ }^{5}$ Oropharyngeal wash specimens were collected by giving each participant $10 \mathrm{ml}$ sterile physiologic saline $(0.9 \%)$ and asked him to gargle the throat for 1 min, then fluids were recollected in $50 \mathrm{ml}$ sterile screw capped cups. ${ }^{5,6}$ In the same time, "nasal swab specimens were obtained using a sterile saline-moistened cotton-tipped plastic swab, which was inserted $\sim 4-5$ $\mathrm{cm}$ into one nostril at an oblique angle toward the turbinate (avoiding the nasopharynx), rotated when resistance was found, extracted, and placed into a tube containing $0.5 \mathrm{~mL}$ of sterile saline". ${ }^{5}$ Collected oral washings were centrifuged at 2,900 $\mathrm{x}$ g for $10 \mathrm{~min}$. Tubes containing nasal swabs were vortexed for $5 \mathrm{~min}$, then each tube was mixed with 2-3 $\mathrm{ml}$ sediment in the corresponding centrifuged tubes with oral washings. The mixed tubes with the two samples were re-centrifuged at 2,900 $\mathrm{x}$ g for $10 \mathrm{~min}$, and then the supernatant was discarded. About $1 \mathrm{ml}$ of sediment was left and frozen at $-80^{\circ} \mathrm{C}$ until the use

\section{DNA extraction and detection}

P. jirovecii DNA was extracted using the QIAmp DNA Mini kit (Qiagen, Hilden, Germany), according to the manufacturer's instructions. One negative control was included in each extraction procedure. Pneumocystis DNA was identified by real-time polymerase chain reaction (PCR) procedure using well designed $P$. jirovecii target gene MSG primers described previously; ${ }^{7-9}$ MSG-fw, GAA TGC AAA TCC TTA CAG ACA ACA G, and MSG-rv, AAA TCA TGA ACG AAA TAA CCA TTG C. These primers are specific for a highly conserved region of human $P$. carinii ( $P$. jirovecii) MSG genes. ${ }^{7}$ The real-time PCR reaction master mixture consisted of $12 \mathrm{uL}$ of Green Star Master Mix (Bioneer, Republic of Korea), 10 pmol each primer $(1 \mu \mathrm{L}), 5 \mu \mathrm{L}$ template DNA, and distilled water to a total volume of 25 $\mu \mathrm{L}$. The PCR reactions were started with a first heat denaturation step at $95^{\circ} \mathrm{C}$ for $10 \mathrm{~min}$, followed by 40 cycles at $95^{\circ} \mathrm{C}$ for $5 \mathrm{sec}$ and $55^{\circ} \mathrm{C}$ for $30 \mathrm{sec}$ on Exicycler 96 real-time PCR system (Bioneer, Republic of Korea). For each PCR run, negative (ultrapure distilled water) and positive (DNA of $P$. jirovecii) controls were included.

\section{Statistical analysis}

Statistical analyses were performed using SPSS (V.15.0). Categorial variables were compared using chi-square test. Continuous variables were compared using Mann-whitney U test. $P$-value $<0.05$ was considered statistically significant.

\section{Results}

The mean of ages was $42.0 \pm 12.5$ years (range 18-75) in general adult group, 32.6 \pm 8.42 years (range 21-60) in health care workers and $58.1 \pm 16.0$ years (range19-83) in immunocompromised individuals. The distribution of sex was as follow; 56 female and 44 male in general adult population, 79 female and 21 male in health care workers, and 23 female and 27 male in immunocompromised individuals. P. jirovecii MSG gene DNA was detected in 57 (22.8\%) of all participants, and $21(21 \%)$ of general adult participants, $22(22 \%)$ of health care workers, $14(28 \%)$ of immunocompromised individuals. Although the frequency of colonization in immunocompromised individuals was higher than other participants, it was not significant statistically $(p=0.610)$. The characteristics of participant and their relations with $P$. jirovecii colonization have been presented in Table 1. Evaluating these characteristics, we found that were no statistical differences in terms of P.jirovecii colonization even though colonization rate higher among the participants applied antibiotic or immunosuppressive treatment (Table 1). Health care workers colonized with $P$. jirovecii were also evaluated according to their occupations (10 of 56 nurses, 8 of 31 physicians and 4 of 13 health care employees), working departments (10 of 40 in internal medicine, 5 of 24 in hematologyoncology, 4 of 16 in intensive care, 1 of 15 in pediatric, and 2 of 5 in surgery), and working durations (mean $101.25 \pm 26.32$ months in health care workers with PCR positive and mean $66.25 \pm 9.69$ months in PCR negative ones); we found any significant relation for none of them $(\mathrm{p}>0.05)$. Underlying diseases of immunocompromised participants consisted of hematological malignity (acute myeloid or lymphoid leukemia and lymphomas) and others (polycythemia and anemia).

Twelve of 35 participants with hematological malignity and two of 15 participants in other group had a positive real-time PCR results for $P$. jirovecii; any significant relationship was not detected between underlying disease and $P$. jirovecii colonization $(p=0.131)$. In addition hospitalization durations of these individuals did not be effective on the colonization rate. However, the duration of underlying disease was longer in the immunocompromised participants colonized with $P$. jirovecii than non-colonized ones ( $p=0.001)$; it was $2-240$ months in the immunocompromised participants colonized with $P$. jirovecii, whereas 10 days-60 months in non-colonized ones.

Table I The characteristics of participant and their relations with P. jirovecii colonization

\begin{tabular}{|c|c|c|c|c|c|c|c|c|}
\hline \multirow{3}{*}{ Characteristics } & \multicolumn{8}{|c|}{ The Number of the participants for the results of P. Jirovecii Real-Time PCR (\%) } \\
\hline & \multicolumn{2}{|c|}{$\begin{array}{l}\text { General Adult population } \\
(n=100)\end{array}$} & \multicolumn{2}{|c|}{$\begin{array}{l}\text { health care workers } \\
(n=100)\end{array}$} & \multicolumn{2}{|c|}{$\begin{array}{l}\text { Immunocompromised } \\
\text { individuals }(n=50)\end{array}$} & \multicolumn{2}{|c|}{ Total $(n=250)$} \\
\hline & Positive & Negative & Positive & Negative & Positive & Negative & Positive & Negative \\
\hline \multicolumn{9}{|l|}{ Gender } \\
\hline Female & 11 & 45 & 16 & 63 & 7 & 16 & $34(21.5)$ & $124(78.5)$ \\
\hline Male & 10 & 34 & 6 & 15 & 7 & 20 & $23(25.0)$ & $69(75.0)$ \\
\hline $\mathrm{p}^{\#}$ & 0.707 & & 0.413 & & 0.723 & & 0.527 & \\
\hline \multicolumn{9}{|l|}{ Age (year) } \\
\hline$<30$ & 3 & 12 & 12 & 32 & I & 3 & $16(25.4)$ & $47(74.6)$ \\
\hline
\end{tabular}




\begin{tabular}{|c|c|c|c|c|c|c|c|c|}
\hline \multirow{2}{*}{$\begin{array}{l}\text { Characteristics } \\
30-49\end{array}$} & \multicolumn{8}{|c|}{ The Number of the participants for the results of P. Jirovecii Real-Time PCR (\%) } \\
\hline & 15 & 41 & 8 & 44 & 1 & 4 & $24(21.2)$ & $89(78.8)$ \\
\hline \multicolumn{9}{|l|}{$\begin{array}{l}p^{\#} \\
0.819\end{array}$} \\
\hline \multicolumn{9}{|c|}{ Chronic lung disease* } \\
\hline Yes & 2 & 7 & 1 & 5 & 2 & 5 & $5(22.7)$ & $17(77.3)$ \\
\hline No & 19 & 72 & 21 & 73 & 12 & 31 & $52(22.8)$ & $176(77.2)$ \\
\hline$P^{\#}$ & 0.808 & & 0.745 & & 0.514 & & 0.993 & \\
\hline \multicolumn{9}{|c|}{ Antibiotic treatment $* *$} \\
\hline Yes & 4 & 10 & 6 & 17 & 7 & 11 & $17(30.9)$ & $38(69.1)$ \\
\hline No & 17 & 69 & 16 & 61 & 7 & 25 & $40(20.5)$ & I55 (79.5) \\
\hline$p^{\#}$ & 0.453 & & 0.59 & & 0.136 & & 0.105 & \\
\hline \multicolumn{9}{|l|}{ Smoking } \\
\hline Yes & 6 & 24 & 6 & 27 & 3 & 3 & I5 (2I.7) & $54(78.3)$ \\
\hline No & 15 & 55 & 16 & 51 & 11 & 33 & $42(23.2)$ & $139(76.8)$ \\
\hline$p^{\#}$ & 0.872 & & 0.518 & & 0.201 & & 0.805 & \\
\hline \multicolumn{9}{|c|}{ Alcohol intake $(>40 \mathrm{~g} / \mathrm{day})$} \\
\hline Yes & 0 & 4 & 4 & 10 & 1 & 1 & $5(25.0)$ & I5 (75.0) \\
\hline No & 21 & 75 & 18 & 68 & 13 & 35 & $52(22.6)$ & I 78 (77.4) \\
\hline$p^{\#}$ & 0.576 & & 0.522 & & 0.479 & & 0.807 & \\
\hline \multicolumn{9}{|c|}{ Immunosuppressive treatment ${ }^{* * * *}$} \\
\hline Yes & 2 & 3 & 3 & 4 & 13 & 35 & $18(30.0)$ & $42(70.0)$ \\
\hline No & 19 & 76 & 19 & 74 & I & 1 & $39(20.5)$ & $|5|(79.5)$ \\
\hline$p^{\#}$ & 0.282 & & 0.167 & & 0.675 & & 0.127 & \\
\hline Total & 21 & 79 & 22 & 78 & 14 & 36 & 57 & 193 \\
\hline
\end{tabular}

\#Analyzed by Pearson chi square test.

*Chronic bronchitis, emphysema, bronchial asthma.

**In last three months for any reason.

***Corticosteroid or any immunosuppressive drug treatment in last six months for any reason.

\section{Discussion}

Although the diagnosis of PCP can be made by microscopic examination of induced sputum and/or bronchoalveolar lavage (BAL) fluid, oropharyngeal washing specimens are preferred materials because of their obtainable quickly and noninvasively in a wide variety of health-care settings also including healthy colonized persons. ${ }^{5,6,10}$ Besides, in a study evaluating the sensitivity of oral wash specimen, 175 oral washes paired with an induced sputum (positive for Pneumocystis) or a BAL sample were assessed by PCR using both MSG and mtLSU-rRNA primers; it has been reported that the oral wash had an excellent sensitivity and MSG primers had a higher sensitivity than the mtLSU-rRNA primers $(91 \%$ and $75 \%$, respectively). ${ }^{10}$ In this study, we tried to determine the colonization prevalence of $P$. jirovecii among adult population in our region. For increase the sensitivity, we used two different noninvasive respiratory specimens from each participant and real-time PCR method with specific primers to a highly conserved region of $P$. jirovecii. To our knowledge, this is the most extensive study evaluating the $P$. jirovecii colonization among general population including also health care workers and immunocompromised individuals in Turkey. The colonization rate has been found $22.8 \%$ in all of our participants while slightly higher in immunocompromised individuals. Previously $P$. jirovecii colonization has been investigated in some limited regions of our country and similar colonization rates has been reported; Ozmen et al. ${ }^{11}$ reported $21 \%$ colonization rate in BAL or bronchial lavage specimens of 100 patients without HIV or PCP, Ozkoc et al., ${ }^{12}$ reported $18.5 \%$ colonization rate in upper pulmonary tissue specimens of 200 cases undergoing forensic autopsy unrelated with HIV or PCP.

Medrano et al., ${ }^{6}$ obtained oropharyngeal wash samples from 50 persons without underlying lung disease or immunosuppression for 
investigation of $P$. jirovecii carriage in the general normal, healthy population; P. jirovecii DNA was amplified in $24 \%(\mathrm{n}=12)$ of samples by nested PCR targeting the mtLSU-rRNA region. In addition these researchers typed this locus in 10 of the 12 samples and reported that the $P$. jirovecii carriage was detected in $20 \%$ of the participants. ${ }^{6}$ Vargas et al., ${ }^{5}$ found the colonization incidence $12.8 \%$ for single oropharyngeal wash specimen, $10.6 \%$ for single nasal swab specimen and $21.5 \%$ for paired oropharyngeal wash and nasal swab specimens by nested PCR in an older population without immuno compromising conditions. They suggested that the mixing of specimens from different anatomical sites before extraction procedure may be a costeffective approach to increase the diagnostic accuracy of Pneumocystis colonization. ${ }^{5}$ Our colonization results detected from paired mix specimens are compatible with their rates. Various risk factors for Pneumocystis colonization have been reported that for instance, the patients receiving immunosuppressive therapy have an increased risk of colonization. ${ }^{3}$ P. jirovecii colonization rate was reported $25.6 \%$ in oropharyngeal wash specimens of the patients receiving infliximab therapy and was statistically associated with duration of infliximab therapy, use of corticosteroid and use of methotrexate. ${ }^{13}$ Our observation relation with the colonization and the use of corticosteroid or other immunosuppressive drug was similar; while the colonization rate was $30 \%$ in the participants receiving any immunosuppressive drug, $20.5 \%$ in others. Therefore, $P$. jirovecii colonization was more frequent among our immunosuppressive participants (28\%) because of the mostly application of immunosuppressive treatment. In addition, the longer duration of underlying disease in this participant group associated with Pneumocystis colonization; his condition may be explained by longer hospitalization, more application of immunosuppressive drugs, and more exposure to other PCP patients.

Healthcare workers may have occupational exposure to $P$. jirovecii, they may be transiently colonized during clinical interactions with PCP patients and subsequently transmit the infection to other immunocompromised patients. ${ }^{14}$ Previously, Miller et al., ${ }^{15}$ had showed that healthcare workers in close occupational contact with patients who had PCP may have become colonized with Pneumocystis. Similarly, Tipirneni et al. ${ }^{14}$ reported higher mean antibody levels to $\mathrm{MsgC} 1$ in clinical healthcare workers than nonclinical workers. However, the colonization rate in healthcare workers was similar to that of general population ( $22 \%$ to $21 \%$ ) in our study. Most probably, occupational exposure to $P$. jirovecii is uncommon because HIV infection and PCP are relatively rare in our country. The contribution of alcohol or smoking to Pneumocystis colonization is uncertain; although it has been reported that cigarette smokers had a higher risk of Pneumocystis colonization than did nonsmokers in some studies, ${ }^{16}$ other researchers did not detect any difference due to alcohol intake and smoking habit as also seen in our findings. ${ }^{6,14}$

\section{Conclusion}

P.jirovecii colonization is not uncommon in our region. Although the colonization rate was higher among immuno suppressor individuals than non-immuno suppressor ones, this difference was not significant. HIV infection is relatively uncommon in Turkey; P.jirovecii has been known as a cause of morbidity and mortality also in other immuno compromised populations. Although the clinical significance of Pneumocystis colonization or association between Pneumocystis colonization and PCP is unclear, colonized individuals may have the potential to serve as a reservoir for maintenance and transmission of the organism to the susceptible population. Therefore, respiratory precautions or respiratory isolation may require presence the patients with PCP and prevention of P. jirovecii transmission may decrease the incidence of this infection.

\section{Acknowledgement}

This work was supported by Eskisehir Osmangazi University Commission Research Projects (BAP, No. 2014-496).

\section{Statement of competing interests}

I declare that I have no significant competing financial, professional or personal interests that might have influenced the performance or presentation of the work described in this manuscript.

\section{References}

1. Morris A, Lundgren J, Masur H, et al. Current Epidemiology of Pneumocystis Pneumonia. Emerg Infect Dis. 2004;10(10):1713-1720.

2. Stern A, Green H, Paul M, et al. Prophylaxis for Pneumocystis pneumonia (PCP) in non-HIV immunocompromised patients. Cochrane Database Syst Rev. 2014;(10):CD005590.

3. Morris A, Norris KA. Colonization by Pneumocystis jirovecii and its role in disease. Clin Microbiol Rev. 2012;(2):297-317.

4. De Boer MG, Bruijnesteijn van Coppenraet LE, Gaasbeek A, et al. An outbreak of Pneumocystis jiroveci pneumonia with 1 predominant genotype among renal transplant recipients: interhuman transmission or a common environmental source? Clin Infect Dis. 2207;44(9):1143-1149.

5. Vargas SL, Pizarro P, López-Vieyra M, et al. Pneumocystis colonization in older adults and diagnostic yield of single versus paired noninvasive respiratory sampling. Clin Infect Dis. 2010;50(3):19-21.

6. Medrano FJ, Montes-Cano M, Conde M, et al. Pneumocystis jirovecii in general population. Emerg Infect Dis. 2205;11(2):245-250.

7. Huang SN, Fischer SH, O'Shaughnessy E, et al. Development of a PCR assay for diagnosis of Pneumocystis carinii pneumonia based on amplification of the multicopy major surface glycoprotein gene family. Diagn Microbiol Infect Dis. 1999;35(1):27-32.

8. Dalpke AH, Hofko M, Zimmermann S. Development and evaluation of a real-time PCR assay for detection of Pneumocystis jirovecii on the fully automated BD MAX platform. J Clin Microbiol. 2013;51(7):2337-2343.

9. Larsen HH, Masur H, Kovacs JA, et al. Development and evaluation of a quantitative, touch-down, real-time PCR assay for diagnosing Pneumocystis carinii pneumonia. J Clin Microbiol. 2002;40(2):490-494.

10. Fischer S, Gill VJ, Kovacs J, et al. The use of oral washes to diagnose Pneumocystis carinii pneumonia: a blinded prospective study using a polymerase chain reaction-based detection system. J Infect Dis. 2001;184(11):1485-1488.

11. Özmen A, Mistık R, Alver O, et al. The Pneumocystis jirovecii colonization in bronchoalveolar lavage (BAL) and bronchial washing and the comparison of methods which are used in diagnosis. Tuberk Toraks. 2013;61(4):303-311.

12. Ozkoc S, Koker M, Onder M, et al. Prevalence of Pneumocystis jirovecii colonization in autopsy cases in Turkey. $J$ Med Microbiol. 2016;65(10):1152-1157.

13. Wissmann G, Morilla R, Martín-Garrido I, et al. Pneumocystis jirovecii colonization in patients treated with infliximab. Eur $J$ Clin Invest. 2011;41(3):343-348. 
14. Tipirneni R, Daly KR, Jarlsberg LG, et al. Healthcare worker occupation and immune response to Pneumocystis jirovecii. Emerg Infect Dis. 2009;15(10): 1590-1597.

15. Miller RF, Ambrose HE, Wakefield AE. Pneumocystis carinii f. sp. hominis DNA in immunocompetent health care workers in contact with patients with P. carinii pneumonia. J Clin Microbiol. 2001;39(11):3877-3882.
16. Morris A, Wei K, Afshar K, et al. Epidemiology and clinical significance of pneumocystis colonization. J Infect Dis. 2008;197(1):10-17. 